\title{
Robust optimal Robin boundary control for the transient heat equation with random input data
}

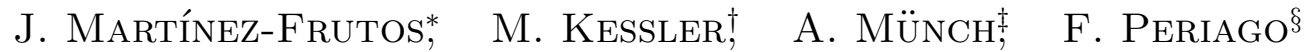

May 4, 2015

\begin{abstract}
The problem of robust optimal Robin boundary control for a parabolic partial differential equation with uncertain input data is considered. As a measure of robustness, the variance of the random system response is included in two different cost functionals. Uncertainties in both the underlying state equation and the control variable are quantified through random fields. The paper is mainly concerned with the numerical resolution of the problem. To this end, a gradient method is proposed. A stochastic collocation finite element method is used for the numerical resolution of the associated state and adjoint state equations. Two numerical experiments illustrate the performance of the algorithm.
\end{abstract}

Keywords: Stochastic heat equation; robust optimal control; random fields; stochastic collocation finite element method.

\section{Introduction}

Physical or engineering systems are always affected by uncertainties that can lead to significant differences between the real systems response and the corresponding numerical models. In particular heat transfer and mass transfer processes are subject to many sources of uncertainty such as random ambient temperature, random initial temperature, random material properties, random heat transfer coefficient or random geometry to name a few. We refer the reader to [5] for a review of the research done in this field. Among the previously mentioned possibly random input parameters, the convective heat transfer coefficient is prevalent because its value strongly depends on random factors such as the motion of the surrounding fluid or the properties of the boundary surface [4, 8].

In the last decade, the presence of unavoidable uncertainties in real-world applications has sparked a number of studies that aim to characterize and control the variability of the final system response. In many optimal control applications the controls act on a system in order to reach an optimal behaviour of the system response. The spatial fluctuations of the PDE input data can

\footnotetext{
* Departamento de Estructuras y Construcción, Universidad Politécnica de Cartagena (UPCT), Campus Muralla del Mar, 30202 Cartagena, Spain - jesus.martinez@upct.es

†Departamento de Matemática Aplicada y Estadística, Universidad Politécnica de Cartagena (UPCT), Campus Muralla del Mar, 30202 Cartagena, Spain

${ }^{\ddagger}$ Laboratoire de Mathématiques de Clermont-Ferrand, UMR CNRS 6620, Campus des Cézeaux 63177 Aubière France - arnaud.munch@math.univ-bpclermont.fr

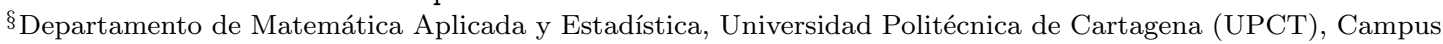
Muralla del Mar, 30202 Cartagena, Spain. E-mail: f.periago@upct.es. Supported by project MTM2013-47053-P from Ministerio de Economía y Competitividad (Spain)
} 
lead to significant changes in the temperature distribution and result in divergence from the target imposed in the deterministic optimal control problem. Therefore, uncertainties associated to the input data should be included in the optimal control formulation. From a mathematical point of view, most of this type of problems are formulated as the minimization of a cost functional subject to a set of constraint equations that appear in the form of partial differential equations (PDEs) with random input data. In addition, the optimal control to be computed should be robust with respect to different realizations of the random input parameters that account for uncertainties in the system. This means that cost functionals should contain not only the mean of the desired quantity to be minimized but also measures of dispersion (e.g, variance, semi-variance and/or others).

The topic of robust optimal control for PDE systems with random input data is an emergent one in the literature. For the case of elliptic PDEs we mention [3, 15] and the references therein. Probably because of the well-known curse of dimensionality phenomenon in the numerical resolution of parametric PDEs, a one-shot approach is proposed in these two works (in contrast with descent methods that require iterations) and the resulting first-order optimality systems are solved by using stochastic collocation and Galerkin finite element methods. More recently, a gradientbased algorithm has been applied for the numerical resolution of a robust optimal design problem for an elliptic PDE with uncertainty in the coefficients and in the boundary conditions [12. The case of parabolic optimal control problems with random coefficients has been hardly studied (we quote [2], where the underlying optimality system is solved by a combination of space-time multigrid methods with sparse-grid collocation techniques). This may partly be due to the fact that up to very recently, there were no efficient methods for solving parabolic PDEs with random input data. In this respect, we mention the important contributions [7, 14, 20.

Another class of control problems that have received a lot of attention in the literature is the controllability for deterministic PDE systems. Its random version, that is, the topic of controllability for PDEs with random coefficients is in its infancy and very little is known in this emergent field. More precisely, the concept of averaged controllability has been recently introduced in [21] and very partial positive (averaged) controllability results have been obtained in [9, 11] for the case of the heat, wave and Schrödinger equations.

The motivation for this paper is twofold: first, we aim at exploring the numerical resolution of robust optimal control problems for a parabolic PDE with random input data by using a descent method. The underlying direct and adjoint systems are solved with a Gear-scheme for the time variable coupled with a stochastic collocation method in the random domain and finite elements in the physical space. Second, a robust optimal control approach for the minimization of the averaged output of the random heat equation is studied. This formulation is more flexible than controllability itself and easily allows to introduce constraints and robustness in the control variable that are difficult to deal with when the controllability problem is directly addressed.

The rest of the paper is organized as follows. Section 2 presents the model problem: a robust optimal Robin boundary control problem for the linear heat equation with uncertainties in the thermal conductivity coefficient, in the convective heat transfer coefficient, in the initial datum and in the control variable. These sources of uncertainty are modelled through random fields. Section 3 is concerned with the numerical resolution of the problem. A gradient method with optimal step-size parameter is proposed. Section 4 presents two numerical experiments in 2D. A final section with concluding remarks closes the paper. 


\section{Problem statement}

\section{$2.1 \quad$ State law}

Let $D \subset \mathbb{R}^{d}, d \geq 1$, be a bounded domain with a Lipschitz boundary $\partial D$, which is decomposed into two disjoint parts $\partial D=\partial D_{0} \cup \partial D_{1}$ (with $\partial D_{0} \cap \partial D_{1}=\emptyset$ ) and let $(\Omega, \mathcal{F}, P$ ) be a complete probability space. Here $\Omega$ denotes the set of outcomes, $\mathcal{F} \subset 2^{\Omega}$ is the $\sigma$-algebra of events and $P: \mathcal{F} \rightarrow[0,1]$ is a probability measure.

Consider the stochastic linear parabolic problem:

$$
\begin{cases}z_{t}(t, x, \omega)-\nabla_{x} \cdot[\kappa(x, \omega) \nabla z(t, x, \omega)]=0 & (t, x, \omega) \in(0, T) \times D \times \Omega \\ z(0, x, \omega)=z^{0}(x, \omega) & (x, \omega) \in D \times \Omega \\ \kappa(y, \omega) \nabla_{x} z(t, y, \omega) \cdot n=0 & (t, y, \omega) \in(0, T) \times \partial D_{0} \times \Omega \\ \kappa(y, \omega) \nabla_{x} z(t, y, \omega) \cdot n=\alpha(u(t, y, \omega)-z(t, y, \omega)) & (t, y, \omega) \in(0, T) \times \partial D_{1} \times \Omega\end{cases}
$$

where the random output $z:(0, T) \times D \times \Omega \rightarrow \mathbb{R}$ is the quantity of interest. The operator $\nabla_{x}$ involves only derivatives with respect to the spatial variable $x \in D$. As usual $n$ stands for the unit outward normal vector to $\partial D$. In practice, the random fields modelling uncertainty in the thermal conductivity coefficient $\kappa$, in the convective heat transfer coefficient $\alpha$, and in the initial datum $z^{0}$ are seldom related and it is therefore reasonable to assume that they are independent. In this sense, $(\Omega, \mathcal{F}, P)$ is the product of the corresponding probability spaces.

\subsubsection{Main Assumptions.}

In order for problem (1) to be well-posed, the following assumptions on the input data are considered:

(A1) $\kappa=\kappa(x, \omega) \in L_{P}^{2}\left(\Omega ; L^{2}(D)\right)$ and there exists $\kappa_{\min }>0$ such that

$$
0<\kappa_{\min } \leq \kappa(x, \omega) \quad \text { a. s. } \omega \in \Omega \text { and a. e. } x \in D .
$$

(A2) $\alpha=\alpha(y, \omega) \in L_{P}^{2}\left(\Omega ; L^{2}\left(\partial D_{1}\right)\right)$ with $\alpha(y, \omega) \geq 0$, there exists a positive constant $\alpha_{\max }<\infty$ such that $\alpha(y, \omega) \leq \alpha_{\max }$ a. s. $\omega \in \Omega$ and a. e. $y \in \partial D_{1}$, and in addition,

$$
\int_{\Omega} \int_{\partial D_{1}} \alpha(y, \omega) d s d P(\omega)>0
$$

where $d s$ is the Lebesgue measure on $\partial D_{1}$.

(A3) $u=u(t, y, \omega) \in L^{2}\left(0, T ; L_{P}^{2}\left(\Omega ; L^{2}\left(\partial D_{1}\right)\right)\right)$

(A4) $z^{0}=z^{0}(x, \omega) \in L_{P}^{2}\left(\Omega ; L^{2}(D)\right)$.

Condition (2) is satisfied with a truncated lognormal Karhunen-Loève expansion of a secondorder random field of the form

$$
\kappa(x, \omega)-\kappa_{0}(x)=\exp \left(\sum_{n=1}^{N} \sqrt{\lambda_{n}} b_{n}(x) Y_{n}(\omega)\right),
$$

with $\kappa_{0} \in L^{\infty}(D)$ such that $\kappa_{0}(x) \geq \kappa_{\min }>0$ a.e. $x \in D$ and the random variables $Y_{n}$ have zero mean, unit variance and are pairwise uncorrelated. If the random field is Gaussian, then $Y_{n} \sim N(0,1)$ are independent and identically distributed (i.i.d.) standard Gaussian variables. 
Remark 1 Due to the upper uniform boundedness condition imposed on $\alpha$, Gaussian variables cannot be considered as a source of uncertainty in this coefficient. However, this is a difficulty only at the theoretical level. Indeed, in practice, a Gaussian variable may be numerically approximated with accuracy by considering its truncation at some large enough lower and upper points. A similar reasoning applies when the coefficient $\kappa_{0}(x)$ which appears in (4) is equal to zero. In this case, if the covariance function of the random field is continuous in $\bar{D} \times \bar{D}$, then the functions $b_{n}(x)$ are continuous in $\bar{D} \times \bar{D}$. Consequently, condition (2) is satisfied and, in addition, there exists a positive constant $\kappa_{\max }$ such that $\kappa(x, \omega) \leq \kappa_{\max }$ a. $s . \omega \in \Omega$ and a. e. $x \in D$.

From a physical point of view, the coefficient $\alpha$ is the most difficult to characterize. Therefore, it is reasonable to use uniformly random variables for the mathematical representation of uncertainty in $\alpha$. As for $\kappa$, the uniform ellipticity condition $(2)$ is physically quite reasonable to assume (see [5. p. 252]).

\subsubsection{Functional Setting.}

The concept of a weak solution of a parabolic problem of the type (1), as considered in [14, 20, is not well suited in optimal control theory where the adjoint state is used as a test function. The asymmetry between the space of solutions and the space of test functions should therefore be corrected. To this end, the following functional setting is considered.

Let $V=H^{1}(D)$ denote the usual Sobolev space and consider the Hilbert space $V_{P}=L_{P}^{2}(\Omega ; V)$ endowed with norm

$$
\|v\|_{V_{P}}=\left(\int_{\Omega} \int_{D}\left(v^{2}+|\nabla v|^{2}\right) d x d P(\omega)\right)^{1 / 2}
$$

The following subspace of $V_{P}$ will play an essential role in the sequel:

$$
V_{P, \kappa}=\left\{v \in V_{P}:\|v\|_{V_{P, \kappa}}^{2}=\int_{\Omega} \int_{D}\left(v^{2}+\kappa|\nabla v|^{2}\right) d x d P(\omega)<\infty\right\} .
$$

Due to the uniform ellipticity condition (2), the space $V_{P, \kappa}$ is continuously embedded in $V_{P}$ and the estimate

$$
\|v\|_{V_{P}} \leq \frac{1}{\sqrt{\kappa_{\min }}}\|v\|_{V_{P, \kappa},}, \quad v \in V_{P, \kappa}
$$

holds.

The dual space of $V_{P, \kappa}$ is denoted by $V_{P, \kappa}^{\star}$. Finally, consider the space

$$
W(0, T)=\left\{z \in L^{2}\left(0, T ; V_{P, \kappa}\right): z_{t} \in L^{2}\left(0, T ; V_{P, \kappa}^{\star}\right)\right\}
$$

endowed with the norm

$$
\|z\|_{W(0, T)}=\left(\int_{0}^{T}\left(\|z(t)\|_{V_{P, \kappa}}^{2}+\left\|z_{t}(t)\right\|_{V_{P, \kappa}^{\star}}^{2}\right) d t\right)^{1 / 2} .
$$

We are now in a position to prove the well-posedness of the state law (1) in this functional framework.

\subsubsection{Well-posedness of (1)}

Definition 2.1 A random function $z=z(t, x, \omega) \in W(0, T)$ is a solution of (1) if it satisfies

$$
\begin{aligned}
\int_{0}^{T}\left(z_{t}, v\right)_{V_{P, \kappa}^{\star}, V_{P, \kappa}} d t & +\int_{0}^{T} \int_{\Omega} \int_{D} \kappa \nabla_{x} z \cdot \nabla_{x} v d x d P(\omega) d t \\
& +\int_{0}^{T} \int_{\Omega} \int_{\partial D_{1}} \alpha z v d s d P(\omega) d t \\
& =\int_{0}^{T} \int_{\Omega} \int_{\partial D_{1}} \alpha u v d s d P(\omega) d t
\end{aligned}
$$


for all $v \in L^{2}\left(0, T ; V_{P, \kappa}\right)$ and where $(\cdot, \cdot)_{V_{P, \kappa}^{\star}, V_{P, \kappa}}$ is the duality product between $V_{P, \kappa}$ and $V_{P, \kappa}^{\star}$. The initial condition $z(0, x, \omega)=z^{0}(x, \omega)$ must be satisfied in $L_{P}^{2}\left(\Omega ; L^{2}(D)\right)$.

Notice that the space $W(0, T)$ is continuously embedded in $C\left([0, T] ; L_{P}^{2}\left(\Omega ; L^{2}(D)\right)\right)$ so that the above initial condition makes sense.

Proposition 2.1 Under assumptions (A1)-(A4) above, there exists a unique solution of (1). Moreover, there exits $c>0$ such that

$$
\|z\|_{W(0, T)} \leq c\left(\left\|z^{0}\right\|_{L_{P}^{2}\left(\Omega ; L^{2}(D)\right)}+\|u\|_{L^{2}\left(0, T ; L_{P}^{2}\left(\Omega ; L^{2}\left(\partial D_{1}\right)\right)\right)}\right) .
$$

Proof. We shall apply the abstract result [19, Th. 26.1]. To this end, consider the Hilbert space $H=L_{P}^{2}\left(\Omega ; L^{2}(D)\right)$ and the Gelfand triple $V_{P, \kappa} \hookrightarrow H \hookrightarrow V_{P, \kappa}^{\star}$. All we need to prove is that the bilinear form $a: V_{P, \kappa} \times V_{P, \kappa} \rightarrow \mathbb{R}$ defined as

$$
a(z, v)=\int_{\Omega} \int_{D} \kappa(x, \omega) \nabla_{x} z \cdot \nabla_{x} v d x d P(\omega)+\int_{\Omega} \int_{\partial D_{1}} \alpha(y, \omega) z v d s d P(\omega)
$$

is continuous and $V_{P, \kappa}$-elliptic, and that the linear form

$$
\begin{aligned}
l: V_{P, \kappa} & \rightarrow \mathbb{R} \\
v & \mapsto l(v)=\int_{\Omega} \int_{\partial D_{1}} \alpha u v d s d P(\omega)
\end{aligned}
$$

is continuous for a. e. $t \in(0, T)$.

The continuity of $a(\cdot, \cdot)$ is an immediate consequence of the Cauchy-Schwarz inequality, the uniform upper bound imposed on $\alpha$ in Assumption (A2), and the continuity of the trace operator. $V_{P, \kappa}$-ellipticity of $a(\cdot, \cdot)$ is a consequence of $(3)$ and may be proved as in the deterministic case (see [18, p. 36]). Finally, the continuity of $l$ follows from assumption (A4).

\subsection{The set of admissible controls}

The considered set of admissible controls is

$$
\mathcal{U}_{a d}=H_{0}^{1}\left(0, T ; L^{2}\left(\partial D_{1}\right)\right)
$$

endowed with its usual norm.

The regularity in time imposed on the control $u$ is justified by the fact that for some control problems and for the specific PDE considered in this paper, it is well-known that controls may exhibit a highly oscillatory behaviour [13. A computational simple way to avoid this undesirable phenomenon is by imposing enough regularity on the control variable. Of course, other choices are possible, for instance, $u \in L^{2}\left(0, T ; L^{2}\left(\partial D_{1}\right)\right)$. It is also quite reasonable from a physical point of view imposing pointwise constraints on the control variable.

As indicated in [15, since physical controller devices are affected by uncertainty, it is realistic to decompose the control variable into an unknown (to be computed) deterministic part, and a stochastic component which probability distribution is assumed to be known. The results that follow, both theoretical and numerical, easily extend to cover this more general situation but for clarity of the exposition we consider the set $\mathcal{U}_{a d}$ as defined in $(9)$.

\subsection{Cost functionals}

Assume that both a final time $T>0$ and a desired target $z_{d} \in L^{2}(D)$ are given. Consider firstthe cost functional

$$
\begin{aligned}
J_{1}(u) & =\frac{1}{2} \int_{D} \int_{\Omega}\left|z(T, x, \omega)-z_{d}(x)\right|^{2} d P(\omega) d x \\
& +\frac{\gamma}{2} \int_{D} \operatorname{Var}(z(T, x)) d x \\
& +\frac{\delta}{2} \int_{0}^{T} \int_{\partial D_{1}}\left(|u(t, y)|^{2}+\left|u_{t}(t, y)\right|^{2}\right) d s d t
\end{aligned}
$$


where $\gamma, \delta \geq 0$ are two weighting parameters, and

$$
\operatorname{Var}(z(T, x))=\int_{\Omega} z^{2}(T, x, \omega) d P(\omega)-\left(\int_{\Omega} z(T, x, \omega) d P(\omega)\right)^{2}
$$

is the variance variance of $z$ at time $T$ and position $x \in D$.

The first term in 10 is a measure of the distance between the state variable $z$ at time $T$ and the prescribed target $z_{d}$. The second term is a measure of robustness and plays the role of increasing the effectiveness of the control variable $u$ for different realizations of the random parameter $\omega$. Finally, the third term has the effect of regularizing the control variable.

Secondly, consider the new cost functional

$$
\begin{aligned}
J_{2}(u) & =\int_{D}\left(\int_{\Omega} z(T, x, \omega) d P(\omega)-z_{d}(x)\right)^{2} d x \\
& +\frac{\gamma}{2} \int_{D} \operatorname{Var}(z(T, x)) d x \\
& +\frac{\delta}{2} \int_{0}^{T} \int_{\partial D_{1}}\left(|u(t, y)|^{2}+\left|u_{t}(t, y)\right|^{2}\right) d s d t .
\end{aligned}
$$

There is a subtle difference between $J_{1}$ and $J_{2}$. Indeed, the first term in (11) measures the $L^{2}(D)$-distance between the expectation of $z(T)$ and the target $z_{d}$ whereas the first term in 10 . accounts for the expectation of the $L^{2}(D)$-distance between $z(T)$ and $z_{d}$.

\subsection{The optimization problem and its physical meaning}

With all of these ingredients, the optimal control problem considered is

$$
\text { (P) } \begin{cases}\text { Minimize in } u \in \mathcal{U}_{a d}: & J_{1}(u) \text { or } \quad J_{2}(u) \\ \text { subject to } & z=z(u) \text { solves (1). }\end{cases}
$$

A deterministic version of this problem has been studied in [6, 16. The physical interpretation of problem (P) follows. A body which occupies the spatial domain $D \subset \mathbb{R}^{d}, d=1,2$ or 3 , is considered. An initial temperature $z^{0}$ of the body and its thermal conductivity coefficient $\kappa$ are known, but these parameters are affected by some uncertainty which is modelled by random fields. A part of the boundary of $D$, denoted by $\partial D_{0}$, is insulated whereas the rest of the boundary $\partial D_{1}$ obeys Newton's law of cooling: convective heat transfer between the body and the surroundings is proportional to the difference of temperatures between the body and the surroundings. Hence, the control variable is the outside temperature $u$. For a desired final temperature $z_{d}$, problem $(\mathrm{P})$ (case of cost functional (10) amounts to find the temperature $u$ which must be applied to $\partial D_{1}$ in order to be closer as possible to $z_{d}$ at time $T$. There is uncertainty in this process, the optimal control $u$ is therefore required to be robust with respect to variations of the uncertain parameters.

For the case of the cost functional (11), the difference is that it is the mean temperature of system (1) which aims at the final target $z_{d}$.

Up to the best knowledge of the authors, an existence theory for the solution of problem $(\mathrm{P})$ has not been addressed so far. However, in some specific situations, the classical tools of deterministic optimal control problems apply to robust optimal control problems for PDEs with random input data. This is the case of problem (P) with $\gamma=0$ and $\delta>0$. In that case, using estimate (6) and the continuous embedding $W(0, T) \hookrightarrow C\left([0, T] ; L_{P}^{2}\left(\Omega ; L^{2}(D)\right)\right)$ it is not difficult to prove that the cost functionals $J_{1}$ and $J_{2}$ are continuous. Since $\gamma=0$, they are convex as well. Moreover, for $\delta>0$ the search for an optimum can be restricted to a closed, convex and bounded subset of $\mathcal{U}_{a d}$. Not only the existence, but also the uniqueness of a solution for $(\mathrm{P})$ then follows. 


\section{$3 \quad$ Numerical resolution of the optimization problem (P)}

For the numerical resolution of problem $(\mathrm{P})$ we propose a gradient method with optimal step-size parameter. Next, we compute descent directions for the cost functionals $J_{1}$ and $J_{2}$ and their associated optimal descent parameters.

\subsection{A descent direction}

Proposition 3.1 The directional derivatives of the cost functionals $J_{1}$ and $J_{2}$ as given, respectively, by 10], (11), at each $u \in \mathcal{U}_{a d}$ in the admissible direction $\hat{u} \in H_{0}^{1}\left(0, T ; L^{2}\left(\partial D_{1}\right)\right)$, exist and take the form

$$
\begin{aligned}
\frac{\partial J_{1}(u)}{\partial u} \cdot \hat{u} & =\frac{\partial J_{2}(u)}{\partial u} \cdot \hat{u} \\
& =\int_{0}^{T} \int_{\Omega} \int_{\partial D_{1}} \alpha(y, \omega) p(t, y, \omega) \hat{u}(t, y) d s d P(\omega) d t \\
& +\delta \int_{0}^{T} \int_{\partial D_{1}}\left(u(t, y) \hat{u}(t, y)+u_{t}(t, y) \hat{u}_{t}(t, y)\right) d s d t
\end{aligned}
$$

where $p=p(t, x, \omega)$ solves the backward in time adjoint problem

$$
\begin{cases}p_{t}(t, x, \omega)+\nabla_{x} \cdot[\kappa(x, \omega) \nabla p(t, x, \omega)]=0 & (t, x, \omega) \in(0, T) \times D \times \Omega, \\ \kappa(y, \omega) \nabla_{x} p(t, y, \omega) \cdot n=0 & (t, y, \omega) \in(0, T) \times \partial D_{0} \times \Omega, \\ \kappa(y, \omega) \nabla_{x} p(t, y, \omega) \cdot n+\alpha(y, \omega) p(t, y, \omega)=0 & (t, y, \omega) \in(0, T) \times \partial D_{1} \times \Omega,\end{cases}
$$

which is completed with the final condition

$$
p(T, x, \omega)=\int_{\Omega} z(T, x, \omega) d P(\omega)-z_{d}(x)+\gamma\left(z(T, x, \omega)-\int_{\Omega} z(T, x, \omega) d P(\omega)\right) .
$$

Consequently, a descent direction for both $J_{1}(u)$ and $J_{2}(u)$ is the function $\bar{u} \in H_{0}^{1}\left(0, T ; L^{2}\left(\partial D_{1}\right)\right)$, which is a weak solution of

$$
-\bar{u}_{t t}+\bar{u}=-\left(\int_{\Omega} \alpha p d P(\omega)+\delta u-\delta u_{t t}\right), \quad 0<t<T, y \in \partial D_{1} .
$$

The expression 12 is obtained by using the formal Lagrange method. Indeed, the Lagrangian associated with problem $(\mathrm{P})$, cost functional $J_{1}$, is defined by

$$
\begin{aligned}
\mathcal{L}(z, u, p) & =J_{1}(u) \\
& -\int_{0}^{T} \int_{D} \int_{\Omega}\left(z_{t}-\nabla(\kappa \nabla z)\right) p_{1} d P(\omega) d x d t \\
& -\int_{0}^{T} \int_{\partial D_{1}} \int_{\Omega}(\kappa \nabla z \cdot n+\alpha(z-u)) p_{2} d P(\omega) d \sigma d t,
\end{aligned}
$$

where $p=\left(p_{1}, p_{2}\right)$. As is well-known, equation $\frac{\partial \mathcal{L}}{\partial z}=0$ gives, after some standard computations, the adjoint system 13 together with the final condition 14 . Similarly, condition $\frac{\partial \mathcal{L}}{\partial u} \cdot v=0$ leads to 12 . A completely analogous reasoning applies to the case of the cost functional (11).

The preceding formal computations may be rigorously proved using standard arguments. We refer to [12] for a very similar computation for the conductivity equation.

From $\sqrt{12}$, it follows easily that the function $\hat{u} \in H_{0}^{1}\left(0, T ; L^{2}\left(\partial D_{1}\right)\right)$ weak solution of 15$)$ is a descent direction for both $J_{1}$ and $J_{2}$. 


\subsection{The optimal descent parameter}

The optimal descent parameter $\lambda$ is obtained as the minimum (over $\mathbb{R}_{+}$) of the quadratic function $\lambda \mapsto J_{i}(u+\lambda \hat{u}), i=1,2$. This can be done explicitly. Indeed, let $\hat{z}(t, x, \omega)$ be the solution of

$$
\begin{cases}\hat{z}_{t}(t, x, \omega)-\nabla_{x} \cdot[\kappa(x, \omega) \nabla \hat{z}(t, x, \omega)]=0, & (t, x, \omega) \in(0, T) \times D \times \Omega \\ \hat{z}(0, x, \omega)=0 & (x, \omega) \in D \times \Omega, \\ \kappa(y, \omega) \nabla_{x} \hat{z}(t, y, \omega) \cdot n=0 & (t, y, \omega) \in(0, T) \times \partial D_{0} \times \Omega, \\ \kappa(y, \omega) \nabla_{x} \hat{z}(t, y, \omega) \cdot n=\alpha(y, \omega)(\hat{u}(t, y, \omega)-\hat{z}(t, y, \omega)) & (t, y, \omega) \in(0, T) \times \partial D_{1} \times \Omega .\end{cases}
$$

It is easy to check that $J_{i}(u+\lambda \hat{u})=J_{i}(u)+a_{i} \lambda+b_{i} \lambda^{2}$, where

$$
\begin{gathered}
a_{1} \quad=\int_{D} \int_{\Omega} \hat{z}(T)\left(z(T, x, \omega)-z_{d}(x)\right) d P(\omega) d x \\
\quad+\gamma\left(\int_{D}\left(\int_{\Omega} z(T) d P(\omega)-\int_{\Omega} z(T) d P(\omega) \int_{\Omega} \hat{z}(T) d P(\omega)\right) d x\right) \\
+\delta \int_{0}^{T} \int_{\partial D_{1}}\left(u(t, y) \hat{u}(t, y)+u_{t}(t, y) \hat{u}_{t}(t, y)\right) d s d t \\
b_{1}=\frac{1}{2} \int_{D} \int_{\Omega} \hat{z}^{2}(T) d P(\omega) d x+\frac{\gamma}{2} \int_{D} \operatorname{Var}(\hat{z}(T, x)) d x+\frac{\delta}{2} \int_{0}^{T} \int_{\partial D_{1}}\left(|\hat{u}(t, y)|^{2}+\left|\hat{u}_{t}(t, y)\right|^{2}\right) d s d t \\
a_{2}=\int_{D}\left(\int_{\Omega} z(T, x, \omega) d P(\omega)-z_{d}(x)\right) \int_{\Omega} \hat{z} d P(\omega) d x \\
+\gamma\left(\int_{D}\left(\int_{\Omega} z(T) \hat{z}(T) d P(\omega)-\int_{\Omega} z(T) d P(\omega) \int_{\Omega} \hat{z}(T) d P(\omega)\right) d x\right) \\
+\delta \int_{0}^{T} \int_{\partial D_{1}}\left(u(t, y) \hat{u}(t, y)+u_{t}(t, y) \hat{u}_{t}(t, y)\right) d s d t
\end{gathered}
$$

and

$b_{2}=\frac{1}{2} \int_{D}\left(\int_{\Omega} \hat{z}(T) d P(\omega)\right)^{2} d x+\frac{\gamma}{2} \int_{D} \operatorname{Var}(\hat{z}(T, x)) d x+\frac{\delta}{2} \int_{0}^{T} \int_{\partial D_{1}}\left(|\hat{u}(t, y)|^{2}+\left|\hat{u}_{t}(t, y)\right|^{2}\right) d s d t$.

Once $a_{i}, b_{i}$ are computed, the optimal step is $\lambda_{i}=-\frac{a_{i}}{2 b_{i}}, i=1,2$.

\subsection{Numerical resolution of the state and adjoint state equations}

For the numerical resolution of the involved PDEs we use a stochastic collocation method in the random space $\Omega$ and $P 1$ Lagrange finite elements in the physical domain $D$. As usual, we make the following assumption:

Assumption 3.1 (Finite dimensional noise) All the involved random input data depend on a finite number $\left\{Y_{n}\right\}_{n=1}^{N}$ of real-valued random variables with mean value zero and unit variance.

We refer to [1, 10, 12] for more details concerning the numerical resolution of the state and adjoint equations as well as the numerical approximation of the related statistical quantities of interest for this problem.

\section{Numerical results}

In this section, we consider two numerical examples to illustrate the performance of the proposed algorithm. The examples cover 2D problems with uncertainties acting in several inputs of the PDE. Experiments 1 and 2 address the control problem considering uncertain heat diffusivity coefficient and uncertain convective heat transfer coefficient, respectively. In all the experiments we consider deterministic targets $z_{d}$ and deterministic control functions. 
The numerical experiments treated in this section are characterized by the following common features (see [12] for more details):

- Finite element approximation in the physical domain $D$. A stochastic collocation method is used to numerically solve both the direct and adjoint equations. The stochastic collocation method requires the resolution of a set of uncoupled deterministic sub-problems in several nodes in the stochastic domain. Each sub-problem is discretized in the physical domain through $P 1$ finite elements on a triangular mesh. The maximum allowed element edge length is fixed to $h=0.03$ in all the numerical experiments.

- Time-discretization scheme. A fully implicit backward second order Gear scheme is used for the time discretization of (1). Starting from $z^{(n-1)}$ at time $t^{(n-1)}$ and $z^{(n)}$ at time $t^{(n)}$. Gear scheme gives

$$
z_{t}^{(n+1)} \equiv \frac{\partial z}{\partial t}\left(t^{(n+1)}\right) \simeq \frac{3 z^{(n+1)}-4 z^{(n)}+z^{(n-1)}}{2 d t}
$$

The same scheme has been used for the adjoint problem (13)-(14).

- Uncertainty modelling. The spatial uncertainties acting in the PDE input data are represented by means of random fields which are approximated by using a truncated KarhunenLoève expansion of the form:

$$
U(x, \omega)=\sum_{n=1}^{N} \sqrt{\lambda_{n}} b_{n}(x) Y_{n}(\omega),
$$

where $\lambda_{n}$ and $b_{n}$ are, respectively, the eigenvalues and eigenvectors of the compact and selfadjoint operator

$$
\psi \mapsto \int_{D} C\left(x, x^{\prime}\right) \psi\left(x^{\prime}\right) d x^{\prime}, \quad \psi \in L^{2}(D),
$$

$C\left(x, x^{\prime}\right)$ is a correlation function and $Y_{n}(\omega)$ is a vector of independent random variables with zero mean and unit variance.

To satisfy the ellipticity condition (2), a lognormal random field is considered to model the uncertainty in the thermal diffusivity. More precisely, we consider

$$
F(x, \omega)=\exp (\eta(x)+\xi(x) U(x, \omega)),
$$

where $\eta(x)$ and $\xi(x)$ are the location and scale parameters of the lognormal distribution, that may depend on $x \in D$. $U$ is a Gaussian random field with zero mean, unit variance and the following isotropic squared exponential correlation function:

$$
C\left(x, x^{\prime}\right)=\exp \left[-\sum_{i=1}^{2} \frac{\left(x_{i}-x_{i}^{\prime}\right)^{2}}{l^{2}}\right],
$$

where $x=\left(x_{1}, x_{2}\right), x^{\prime}=\left(x_{1}^{\prime}, x_{2}^{\prime}\right) \in D$, and $l>0$ is the correlation length.

As in [12, the size $N$ of the expansion is set in order to capture $95 \%$ of the energy field.

- Collocation approximation in the stochastic domain $\Omega$. When the random variables $Y_{n}$ come from a Karhunen-Loève expansion of a Gaussian field, they are independent standard Gaussian. Hence, the corresponding collocation nodes $y_{k}$ are the Cartesian products 


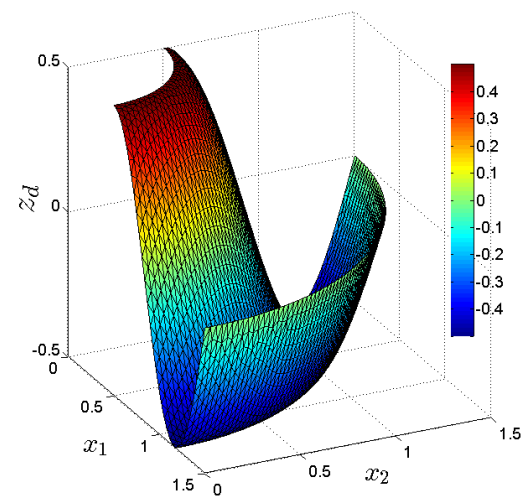

(a)

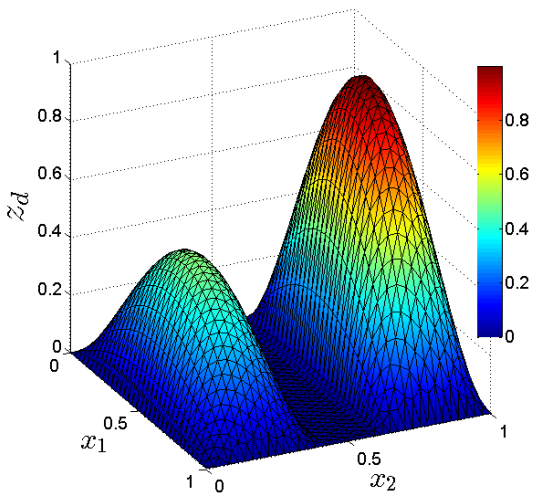

(b)

Figure 1: Deterministic target function $z_{d}$ used in Experiment 1 (a) and in Experiment 2 (b).

determined by the roots of Hermite polynomials. In order to reduce the computational cost related to the full tensor product rules, an isotropic sparse grid, as proposed in [17, has been applied. Given a $1 \mathrm{D}$ quadrature rule $\mathcal{Q}^{k}$ of level $k$, the $M$-dimensional Smolyak isotropic product rule is defined as follows:

$$
\mathcal{A}(k, M)=\sum_{k-M+1 \leq|i| \leq k}(-1)^{k+M-|i|}\left(\begin{array}{c}
k+M \\
k+M-|i|
\end{array}\right)\left(\mathcal{Q}^{i_{1}} \otimes \cdots \otimes \mathcal{Q}^{i_{M}}\right)
$$

The dimension of the product rule is equal to the size $N$ of the Karhunen-Loève expansion. The low degree of intrusiveness of the stochastic collocation method allows to solve the stochastic PDE on parallel computers which reduces the computational cost of the optimization algorithm.

\subsection{Experiment 1: Uncertainty in the thermal diffusivity}

The goal of the first experiment is to check numerically the averaged exact controllability property as introduced in [9, 21, under uncertainty which is quantified through a Gaussian type field acting on the thermal diffusivity.

The spatial domain is

$$
D=\left\{(x=r \cos \theta, y=r \sin \theta) \in \mathbb{R}^{2}: 0.5 \leq r \leq 1.5,0 \leq \theta \leq \pi / 2\right\},
$$

the boundary of the domain is divided into $\partial D_{1}=\left\{(r, \theta) \in \mathbb{R}^{2}: r=1.5\right.$ and $\left.0 \leq \theta \leq \pi / 2\right\}$ and $\partial D_{0}=\partial D \backslash \partial D_{1}$. The initial condition is $z(0, x, \omega)=0$, the final controllability time is $T=0.5$ and the desired target is $z_{d}(r)=0.5 \sin (3 \pi r / 2-\pi / 4)$ in $D$ (Figure 1 1 a).

The space domain is discretized using a finite element mesh composed of $3905 \mathrm{P} 1$ triangular elements and 743 spatial degrees of freedom. The time domain is discretized using the implicit backward second order scheme given by 22 with $d t=2.5 \times 10^{-3}$. The thermal diffusivity $\kappa(x, \omega)$ is represented by a lognormal random field with mean 1.0 and coefficient of variation 0.1 . This lognormal random field is obtained through the transformation (23)-(24) with $\eta=-0.0477$ and $\xi=0.3087$. The Karhunen-Loève expansion 23 is truncated at nine terms. The stochastic collocation in $\Omega$ is performed using a three-level isotropic Smolyak sparse grid based on GaussHermite quadrature rule producing $Q=649$ collocation points. 


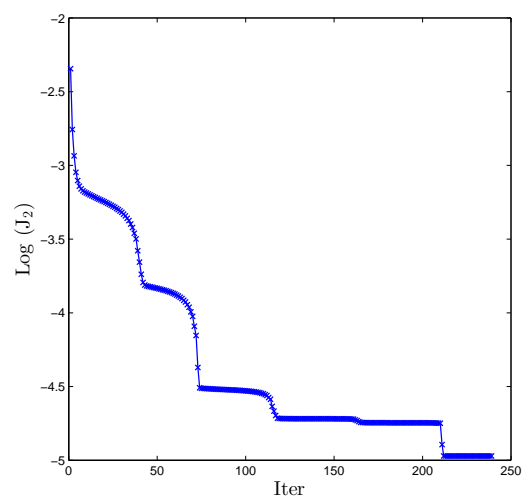

Figure 2: Convergence history of the optimization algorithm for the cost $J_{2}, \gamma=1$ and $\delta=1 e-8$.

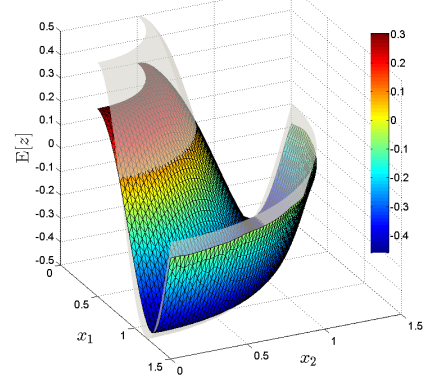

(a)

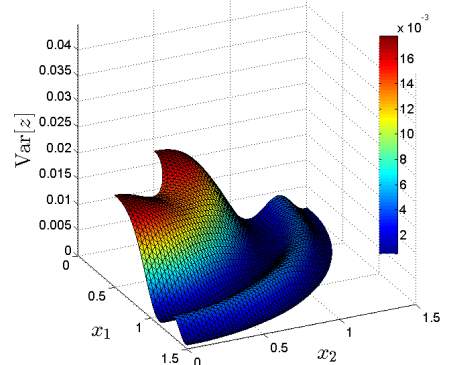

(b)

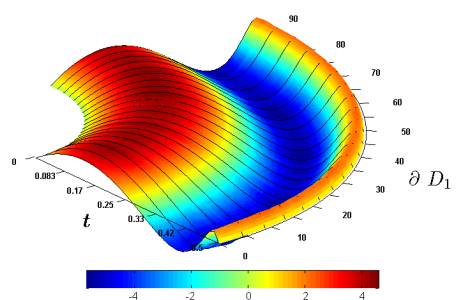

(c)

Figure 3: Results associated with the cost $J_{1}$ for Experiment 1 with $\gamma=0, \delta=1 e-8$. The following quantities are represented: the mean of the state variable (a), the variance of the state variable (b) and the control variable (c). The target $z_{d}$ is shown transparently in (a) as a reference.

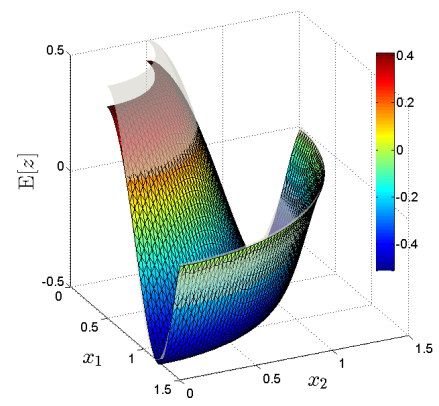

(a)

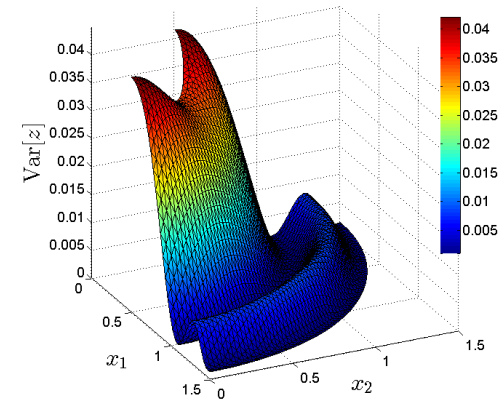

(b)

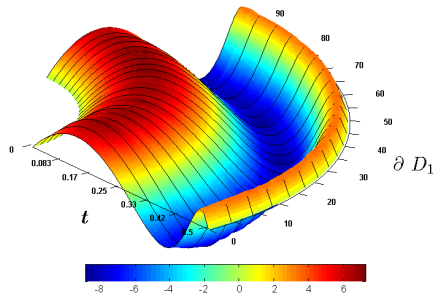

(c)

Figure 4: Results associated with the cost $J_{2}$ for Experiment 1 with $\gamma=0, \delta=1 e-8$. The following quantities are represented: the mean of the state variable (a), the variance of the state variable (b) and the control variable (c). The target $z_{d}$ is shown transparently in (a) as a reference. 


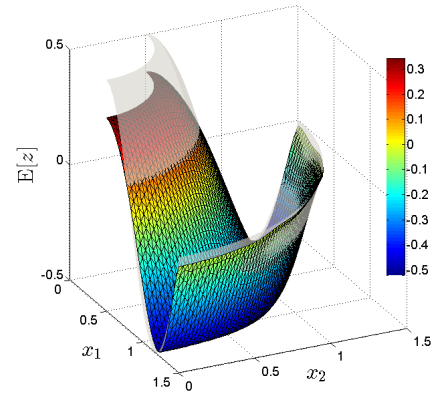

(a)

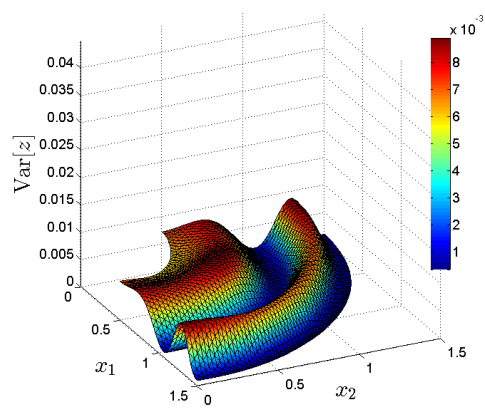

(b)

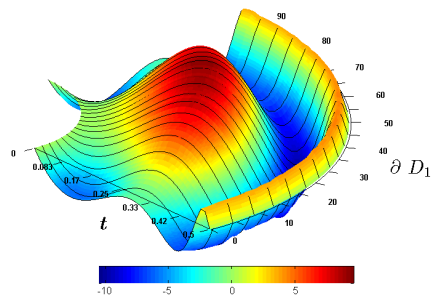

(c)

Figure 5: Results associated with the cost $J_{2}$ for Experiment 1 with $\gamma=1, \delta=1 e-8$. The following quantities are represented: the mean of the state variable (a), the variance of the state variable (b) and the control variable (c). The target $z_{d}$ is shown transparently in (a) as a reference.

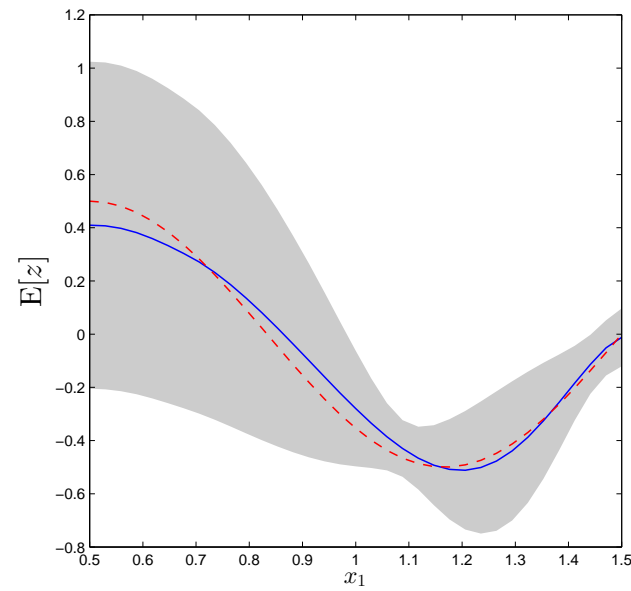

(a)

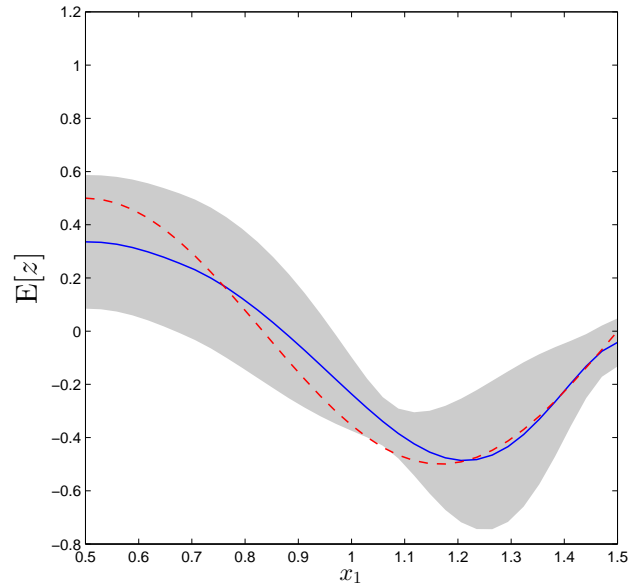

(b)

Figure 6: Experiment 1: correspondence between the stochastic state variable (blue line) and the target (dotted red line) in $x \in[0.5,1.5] \times[0]$. Results obtained for cost $J_{2}, \gamma=0$ (a) and $\gamma=1$ (b).

Table 1: Summary of the error with respect to the target and variance of the state variable for Experiment 1. Here $\bar{z}(T, x)$ denotes the mean of $z(T, x, \omega)$, i.e., $\bar{z}(T, x)=\int_{\Omega} z(T, x, \omega) d P(\omega)$.

\begin{tabular}{ccc}
\hline & $\left\|\bar{z}(T)-z_{d}\right\|_{L^{2}(D)}^{2}$ & $\int_{D} \operatorname{Var}(z(T, x)) d x$ \\
\hline$J_{1}, \gamma=0$ & $6.426 \times 10^{-3}$ & $4.336 \times 10^{-3}$ \\
$J_{1}, \gamma=1$ & $7.626 \times 10^{-3}$ & $3.758 \times 10^{-3}$ \\
$J_{1}, \gamma=2$ & $1.013 \times 10^{-2}$ & $3.059 \times 10^{-3}$ \\
$J_{1}, \gamma=3$ & $1.123 \times 10^{-2}$ & $2.621 \times 10^{-3}$ \\
$J_{2}, \gamma=0$ & $1.238 \times 10^{-3}$ & $8.783 \times 10^{-3}$ \\
$J_{2}, \gamma=1$ & $3.324 \times 10^{-3}$ & $3.542 \times 10^{-3}$ \\
$J_{2}, \gamma=2$ & $5.259 \times 10^{-3}$ & $2.845 \times 10^{-3}$ \\
$J_{2}, \gamma=3$ & $6.639 \times 10^{-3}$ & $2.205 \times 10^{-3}$ \\
\hline
\end{tabular}




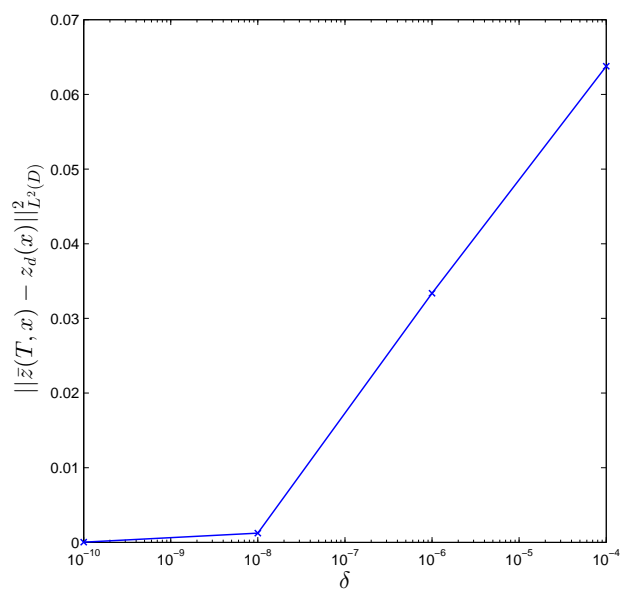

Figure 7: Error with respect to the target $\left\|\bar{z}(T)-z_{d}\right\|_{L^{2}(D)}^{2}$ as a function of the penalty parameter $\delta$ for the numerical experiment 1 with cost $J_{2}$ and $\gamma=0$.

The optimal control problem (P) has been solved for the costs $J_{1}$ and $J_{2}$ and for different values of the weighting parameter $\gamma=0,1,2,3$. The algorithm is initialized with a control $u^{0}=0$, and stops at the first $j \in \mathbb{N}$ for which $\left|J_{i}\left(u^{j}\right)-J_{i}\left(u^{j-1}\right)\right| \leq \epsilon J_{i}\left(u^{j}\right)$ or $\left|\eta^{j}\right| \leq \epsilon, i=1,2$, where $\epsilon=1 e-9$, and $u^{j}, \eta^{j}$ are the control and the descent parameter, respectively, at iteration $j$. The convergence history of the optimization algorithm, for the cost $J_{2}, \gamma=1$ and $\delta=1 e-8$ is shown in log-scale in Figure 2 ,

The $L^{2}$ - norm of the error with respect to the target and the variance of the state variable are summarized in Table 1. The mean and the variance of the state variable and the control profile over time are shown in Figure 3 for cost function $J_{1}, \gamma=0$ and $\delta=1 e-8$. Figure 4 shows the results obtained for the cost $J_{2}$ using the same parameters. Comparing the results in Fig. 3 and 4 it is observed that the minimization of the cost $J_{1}$ provides a solution with lower variance of the state variable at the cost of increasing the error with respect to the target. In both cases, the variance can be reduced by increasing the value of the parameter $\gamma$ as shown in Figure 5 for cost $J_{2}$ and $\gamma=1$. The peak value of the variance is significantly decreased whereas the error with respect to the target increases slightly. This effect is also observed in Figure 6, where the mean of the state variable is shown in blue line whereas the dotted red line represents the target function. The shadow region shows the $99 \%$ confidence interval of $z$. It is observed in Figure $6 \mathrm{~b}$ that the maximum width of the confidence interval is reduced at the cost of worsening the approximation to the target. The reduction in the confidence interval is accompanied by a notably change in the control profile as shown in Figure 6.

The error with respect to the target $\left\|\bar{z}(T)-z_{d}\right\|_{L^{2}(D)}^{2}$ for cost $J_{2}$ and $\gamma=0$ is analyzed for different values of the penalty parameter $\delta$. Figure 7 shows how the error between the mean of state variable and the target function increases when the parameter $\delta$ increases. This error is closed to zero for $\delta=1 e-10$.

\subsection{Experiment 2: Uncertainty in the convective heat transfer coeffi- cient}

A unit square spatial domain $D=] 0,1\left[^{2}\right.$ is considered. Its boundary is divided into $\partial D_{1}=$ $\left\{\left(x_{1}, x_{2}\right) \in \mathbb{R}^{2}: x_{2}=0\right\} \cup\left\{\left(x_{1}, x_{2}\right) \in \mathbb{R}^{2}: x_{2}=1\right\}$ and $\partial D_{0}=\partial D \backslash \partial D_{1}$. The initial condi- 
Table 2: Summary of the error with respect to the target and variance of the state variable for Experiment 2. Here $\bar{z}(T, x)$ denotes the mean of $z(T, x, \omega)$, i.e., $\bar{z}(T, x)=\int_{\Omega} z(T, x, \omega) d P(\omega)$.

\begin{tabular}{ccc}
\hline & $\left\|\bar{z}(T)-z_{d}\right\|_{L^{2}(D)}^{2}$ & $\int_{D} \operatorname{Var}(z(T, x)) d x$ \\
\hline$J_{1}, \gamma=0$ & $6.167 \times 10^{-3}$ & $2.663 \times 10^{-3}$ \\
$J_{1}, \gamma=1$ & $4.796 \times 10^{-3}$ & $1.478 \times 10^{-3}$ \\
$J_{1}, \gamma=2$ & $1.029 \times 10^{-2}$ & $1.318 \times 10^{-3}$ \\
$J_{1}, \gamma=3$ & $1.156 \times 10^{-2}$ & $7.757 \times 10^{-4}$ \\
$J_{2}, \gamma=0$ & $3.380 \times 10^{-3}$ & $3.895 \times 10^{-3}$ \\
$J_{2}, \gamma=1$ & $4.755 \times 10^{-3}$ & $1.588 \times 10^{-3}$ \\
$J_{2}, \gamma=2$ & $6.053 \times 10^{-3}$ & $1.267 \times 10^{-3}$ \\
$J_{2}, \gamma=3$ & $9.472 \times 10^{-3}$ & $7.992 \times 10^{-4}$ \\
\hline
\end{tabular}

tion is $z(0, x, \omega)=0$ and the final control time is $T=0.5$. The following piecewise target function is considered

$$
z_{d}\left(x_{1}, x_{2}\right)= \begin{cases}\left(-2 x_{1}^{2}+2 x_{1}\right)\left(-25 x_{2}^{2}+10 x_{2}\right) & 0 \leq x_{1} \leq 1,0 \leq x_{2} \leq 0.4 \\ 0 & 0 \leq x_{1} \leq 1,0.4 \leq x_{2} \leq 0.6 \\ \left(-4 x_{1}^{2}+4 x_{1}\right)\left(-25 x_{2}^{2}+40 x_{2}-15\right) & 0 \leq x_{1} \leq 1.0,0.6 \leq x_{2} \leq 1\end{cases}
$$

This target function is depicted in Figure $1 \mathrm{p}$. The finite element mesh is composed of $2436 \mathrm{P} 1$ triangular elements and 1310 degrees of freedom. A time step of $d t=2.5 \times 10^{-3}$ is considered in the Gear scheme 22.

The random heat transfer coefficient $\alpha(y, \omega)$ is decomposed into a deterministic part and a zero-mean stochastic part $\alpha(y, \omega)=\bar{\alpha}+\hat{\alpha}(y, \omega)$. The deterministic component $\bar{\alpha}$ is set to 1 whereas the stochastic part $\hat{\alpha}$ is represented by two lognormal random fields with zero mean and variance 0.01 at $x_{2}=0$ and variance 0.1 at $x_{2}=1$. The random fields are discretized by means of a Karhunen-Loève expansion using a exponential covariance function with correlation length of 0.5 and variance 0.01 at $x_{2}=0$ and variance 0.1 at $x_{2}=1$. The Karhunen-Loève expansion is truncated at three terms. The independent random variables of the K-L expansion are assumed to be uniformly distributed on $[-3,3]$. The stochastic collocation in $\Omega$ is performed using a threelevel isotropic Smolyak sparse grid based on Gauss-Legendre quadrature rule producing $Q=85$ collocation points.

For $\gamma=0,1,2,3$, Table 2 collects results for the different contributions of the costs $J_{1}$ and $J_{2}$ after convergence of the algorithm (the criterium of convergence is the same as in the preceding experiment). The upper and lower controls are shown in Figures 8 and Figure 10 for costs $J_{1}$ and $J_{2}$, respectively. As expected the peak value of the variance (Figures $8 \mathrm{p}$ and $10 \mathrm{p}$ ) of the state variable is close to the upper edge due to the larger covariance of the random field on this part of the boundary. Comparing Figures 8 and 10 it is observed that, as in Experiment 1, the cost $J_{2}$ provides a better correspondence between the mean of state variable and the target function but with a larger variance. This variance can be reduced by increasing the value of the parameter $\gamma$ as shown in Figure 11 for $\gamma=1,2$ and 3 .

Figure 9 shows the error between the mean of state variable and the target function as a function of the penalty parameter $\delta$. Contrary to the experiment 1 , in this case the error does not approach to zero as the penalty parameter is reduced. This is due to the fact that the imposed target is not a trajectory of the forward problem and thus cannot be reached.

The first term of the cost $J_{1}$ implicitly penalizes the variance of the state variable and it is 


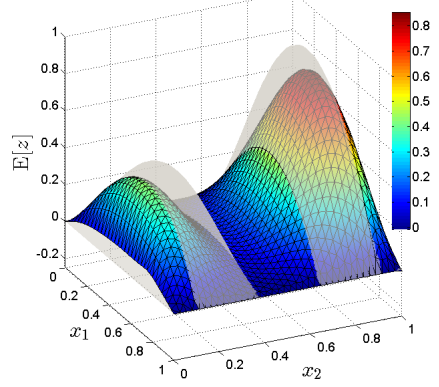

(a)

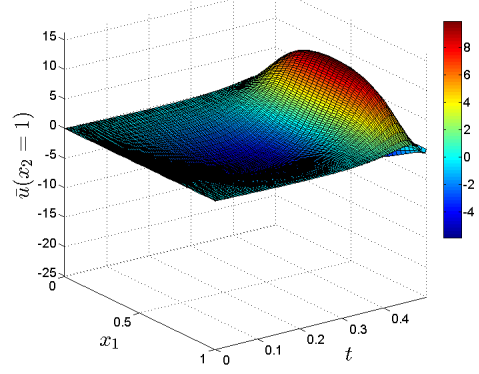

(c)

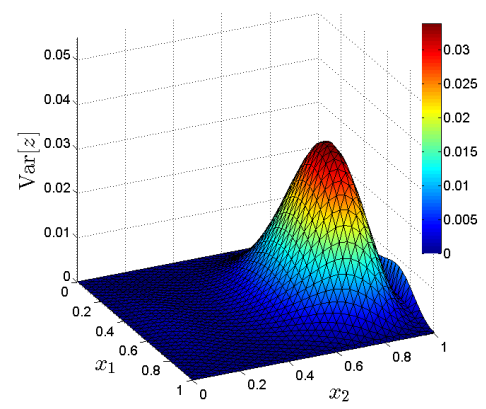

(b)

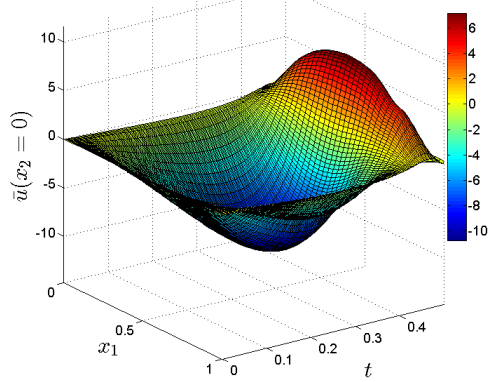

(d)

Figure 8: Results associated with the cost $J_{1}$ for Experiment 2 with $\gamma=0, \delta=1 e-8$. The following quantities are represented: the mean of the state variable (a), the variance of the state variable (b), the control variable acting on $x_{2}=1$ (c) and the control variable acting on $x_{2}=0$. The target $z_{d}$ is shown transparently in (a) as a reference.

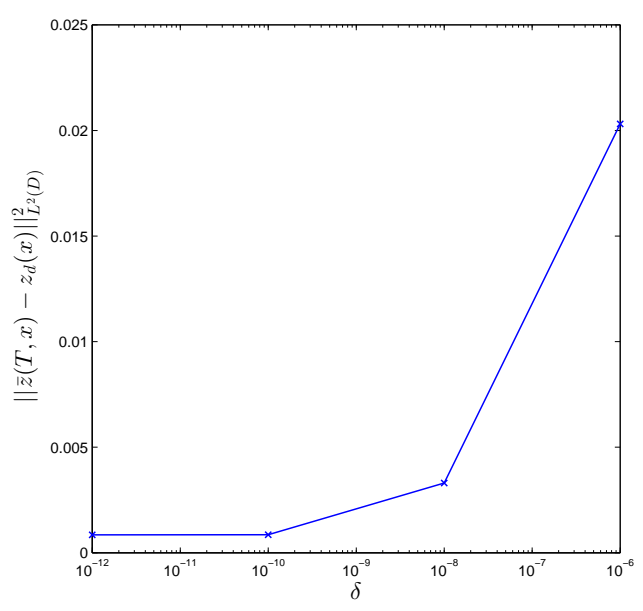

Figure 9: Error with respect to the target $\left\|\bar{z}(T)-z_{d}\right\|_{L^{2}(D)}^{2}$ as a function of the penalty parameter $\delta$ for the numerical experiment 2 with cost $J_{2}$ and $\gamma=0$. 


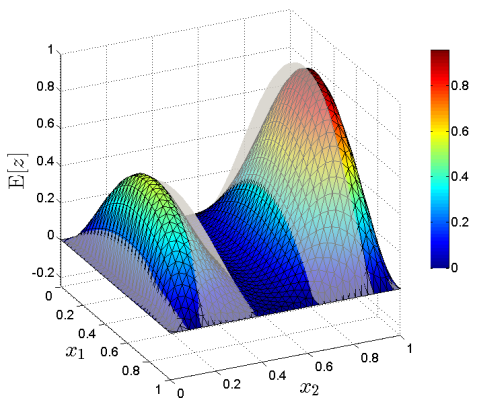

(a)

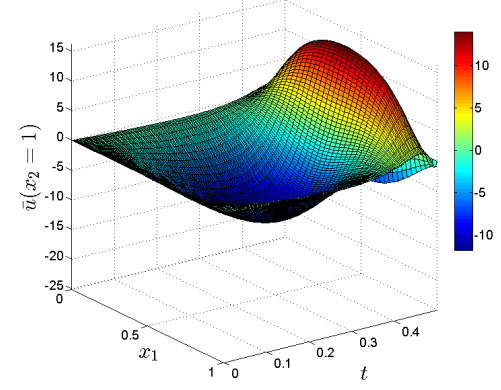

(c)

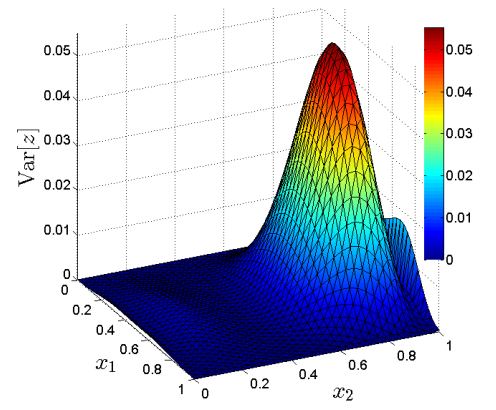

(b)

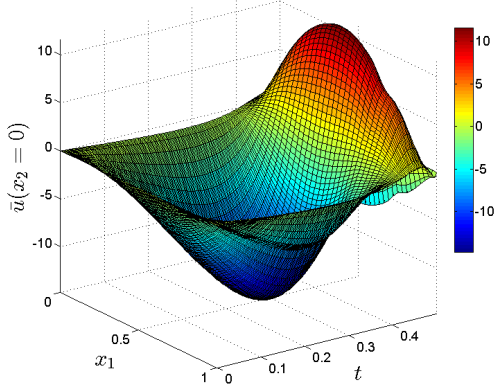

(d)

Figure 10: Results associated with the cost $J_{2}$ for Experiment 2 with $\gamma=0, \delta=1 e-8$. The following quantities are represented: the mean of the state variable (a), the variance of the state variable (b), the control variable acting on $x_{2}=1$ (c) and the control variable acting on $x_{2}=0$. The target $z_{d}$ is shown transparently in (a) as a reference. 


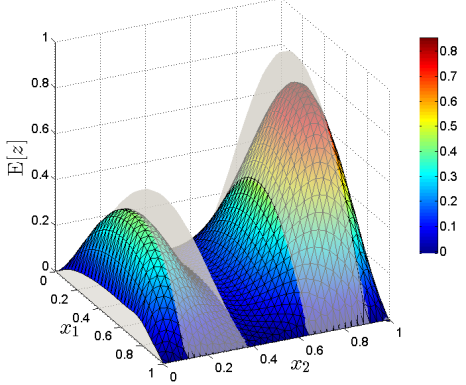

(a)

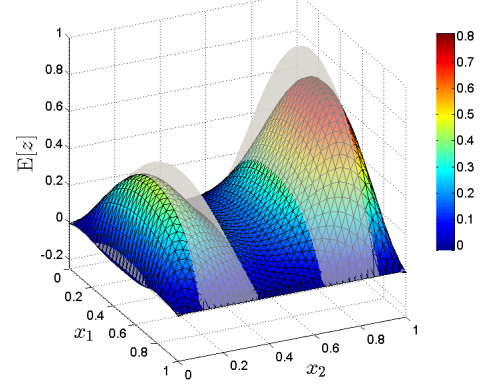

(c)

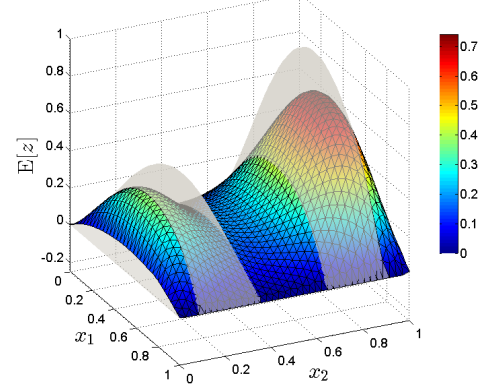

(e)

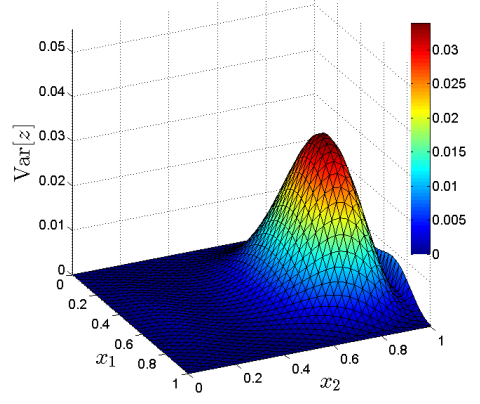

(b)

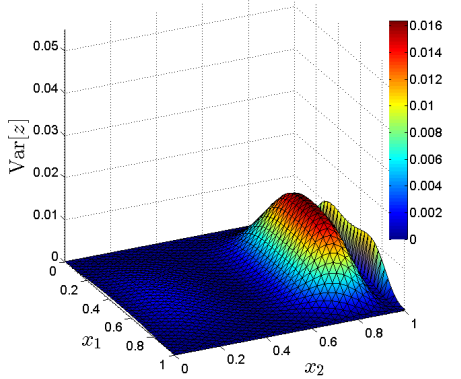

(d)

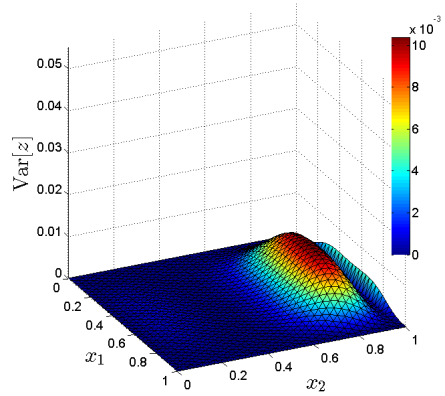

(f)

Figure 11: Results associated with the cost $J_{2}$ for Experiment 2 with $\delta=1 e-8, \gamma=1$ (a,b), $\gamma=2$ $(\mathrm{c}, \mathrm{d})$ and $\gamma=3(\mathrm{e}, \mathrm{f})$. The following quantities are represented: the mean of the state variable on the left-hand side of the panel and the variance of the state variable on the right-hand. The target $z_{d}$ is shown transparently in (a,c,d) for reference. 
therefore not possible to obtain the best approximation to the target. On the contrary, the cost $J_{2}$ provides more flexibility since it allows to isolate the conflicting objectives, namely approximation to the target function and reduction of the state variable variability. The cost $J_{2}$ finds a better trade-off between the two conflicting objectives modifying the parameter $\gamma$.

\section{Conclusion}

A gradient method for a stochastic optimal control problem constrained by the transient heat equation with random coefficients has been presented. Uncertainty in those coefficients is quantified through random fields. The first and second order statistical moments of the system random output have been included in two different cost functionals. A stochastic collocation method has been used to solve the underlying state and adjoint state equations. Since the variance of the system response is included in the cost functional as a measure of robustness, the non-intrusive character of the collocation method is lost. Even so, and taking also into account that a second-order scheme has been used to discretize the time variable, the hardest part of the computations in the gradient method are done on parallel computers in such a way that the iterative optimization algorithm can be solved for a relative large number of terms in the truncated Karhunen-Loève expansion of the random fields that account for uncertainties. As a conclusion, gradient-based optimization algorithms are numerically competitive for solving robust optimal control problems constrained by time-dependent, parametric, PDEs.

The examples considered in the experiments are a first step towards the numerical resolution of the robust, averaged controllability problem for parametric PDEs, which is an emergent topic in the literature.

\section{References}

[1] I. Babuška, F. Nobile, And R. Tempone, A stochastic collocation method for elliptic partial differential equations with random input data, SIAM Review, 52 (2010), pp. 317-355.

[2] A. Borzi AND G. VON Winckel, Multigrid methods and sparse-grid collocation techniques for parabolic optimal control problems with random coefficients, SIAM J. Sci. Comput., 31 (2009), pp. 2172-2192.

[3] P. Chen, A. Quarteroni, And G. Rozza, Stochastic optimal robin boundary control problems of advection-dominated elliptic equations, SIAM J. Numer. Anal., 51 (2013), pp. 27002722 .

[4] R. CHIBA, Stochastic heat conduction analysis of a functionally graded annular disc with spatially random heat transfer coefficients, Appl. Math. Model., 33 (2009), pp. 507-523.

[5] — Stochastic analysis of heat conduction and thermal stresses in solids: a review, 2012, ch. 9, pp. 243-266.

[6] V. Dhamo and F. Tröltzsch, Some aspects of reachability for parabolic boundary control problems with control constraints, Comput. Optim. Appl., 50 (2011), pp. 75-110.

[7] C. Gittelson, R. Andreev, And C. Schwab, Optimality of adaptive galerkin methods for random parabolic partial differential equations, J. Comput. Appl. Math., 263 (2014), pp. 189201. 
[8] A. Kuznetsov, Stochastic modeling of heating of a one-dimensional porous slab by a flow of incompressible fluid, Acta Mech., 114 (1996), pp. 39-50.

[9] M. Lazar and E. Zuazua, Averaged control and observation of parameter-depending wave equations, C. R. MATH., 352 (2014), pp. 497-502.

[10] G. Lord, C. Powell, and T. Shardlow, An Introduction to Computational Stochastic PDEs, 2014.

[11] Q. Lü AND E. ZuAZuA, Averaged controllability for random evolution partial differential equations, arXiv:1501.05102, (2015).

[12] J. Martínez-Frutos, M. Kessler, and F. Periago, Robust optimal shape design for an elliptic pde with uncertainty in its input data, to appear in ESAIM:COCV.

[13] A. MÜnCh AND E. Zuazua, Numerical approximation of null controls for the heat equation : Ill-posedness and remedies, Inverse Probl., 26 (2010), p. 085018.

[14] F. Nobile And R. Tempone, Analysis and implementation issues for the numerical approximation of parabolic equations with random coefficients, Internat. J. Numer. Methods Engrg., 80 (2009), pp. 979-1006.

[15] E. Rosseel And G. Wells, Optimal control with stochastic pde constraints and uncertain controls, Comput. Methods Appl. Mech. Eng., 213-216 (2012), pp. 152-167.

[16] K. SchittKowski, Numerical solution of a time-optimal parabolic boundary-value control problem, J. Optim. Theory Appl., 27 (1979), pp. 271-290.

[17] S. Smolyak, Quadrature and interpolation formulae for tensor products of certain classes of functions, Doklady Akademii Nauk SSSR, 4 (1963), pp. 240-243.

[18] F. Tröltzsch, Optimal Control of Partial Differential Equations: Theory, Methods and Applications, 2010.

[19] J. Wloka, Partial Differential Equations, 1987.

[20] G. Zhang And M. Gunzburger, Error analysis of a stochastic collocation method for parabolic partial differential equations with random input data, SIAM J. Numer. Anal., 50 (2012), pp. 1922-1940.

[21] E. Zuazua, Averaged control, Automatica, 50 (2014), pp. 3077-3087. 